\title{
NOVAS DROGAS NO TRATAMENTO DA DISPEPSIA FUNCIONAL
}

\author{
Luiz Ernesto de Almeida TRONCON*
}

RESUMO - Racional - A dispepsia funcional caracteriza-se pela ocorrência de sintomas digestivos altos, na ausência de qualquer evidência de doença orgânica ou anormalidade estrutural. O tratamento farmacológico atual desta condição é feito empiricamente com agentes anti-secretores ou drogas pró-cinéticas. Objetivos - Rever os avanços recentes nos conhecimentos sobre os mecanismos responsáveis pelo aparecimento dos sintomas, bem como o desenvolvimento de novas drogas capazes de interferir nestes mecanismos, que abrem a perspectiva de tratamento farmacológico mais racional e efetivo da dispepsia funcional. Método - Revisão sistemática do trabalhos publicados em língua inglesa, nos últimos 10 anos. Resultados - Novas drogas que aumentam a propulsão gastroduodenal incluem: derivados benzamídicos análogos da cisaprida, antagonistas dos receptores A da colecistocinina, agonistas de receptores opióides e uma nova classe de agonistas da motilina, semelhantes à eritromicina. Drogas agonistas dos receptores serotoninérgicos do plexo mioentérico, como o sumatriptano e a buspirona, podem aumentar a capacidade de acomodação do bolo alimentar. Por fim, novas drogas, de grupos farmacológicos diversos, estão sendo avaliadas quanto a sua capacidade de reduzir ou modificar a percepção sensorial visceral. Inclui este grupo a octreotida, a loxiglumida, o ondansetron e seus análogos, a fedotozina e os anti-depressivos tricíclicos, em doses baixas. Conclusões - Ainda que as novas drogas apresentem alto potencial de aperfeiçoar o tratamento da dispepsia funcional, há a necessidade de ensaios controlados, com número adequado de pacientes, para se comprovar a eficácia desses medicamentos. Além disso, as dificuldades em determinar o mecanismo subjacente aos sintomas pode limitar o impacto positivo das novas drogas no tratamento da dispepsia funcional.

DESCRITORES - Dispepsia, quimioterapia. Enteropatias, fisiopatologia. Enteropatias, quimioterapia. Quimioterapia. Motilidade gastrointestinal.

\footnotetext{
* Professor Associado, Departamento de Clínica Médica (Divisão de Gastroenterologia), Faculdade de Medicina de Ribeirão Preto, Universidade de São Paulo, Ribeirão Preto, SP.

Endereço para correspondência: Dr. Luiz E. A. Troncon - Departamento de Clínica Médica Hospital das Clínicas - Campus da USP - 14048-900 - Ribeirão Preto, SP. e-mail ledatron@fmrp.usp.br
} 


\section{INTRODUÇÃO}

A dispepsia funcional é uma condição clínica que se caracteriza pela ocorrência de sintomas atribuíveis aos segmentos mais proximais do trato gastrointestinal em pacientes que não apresentam qualquer evidência de doença orgânica ou anormalidade estrutural do tubo digestivo, mesmo após adequada investigação endoscópica, ultrasonográfica, bioquímica, hematológica e microbiológica ${ }^{(2,14,26,40,41)}$. A dispepsia funcional integra um conjunto variado de doenças funcionais digestivas que, a partir da década de 1980, tem sido sistematizado quanto aos critérios diagnósticos por comitês de especialistas que se reuniram pela primeira vez em Roma, Itália ("Critérios de Roma")(41). É condição clínica muito comum em todo o mundo, constituindo o mais importante dos distúrbios funcionais gastroduodenais ${ }^{(41)}$.

Sua etiologia, bem como os mecanismos envolvidos na produção dos sintomas nesta entidade, não são perfeitamente conhecidos ${ }^{(2,14}$, 26). Assim sendo, o seu tratamento é, em grande parte, de natureza empírica. Abrange medidas de ordem geral e o uso de medicamentos que, agindo em pontos incertos da provável fisiopatologia da doença, provocam, em grau variável e imprevisível, o alívio dos sintomas ${ }^{(26,}$ $39,46)$

Nos últimos anos, diversos estudos têm produzindo informações que vêm aumentando, de modo considerável, o entendimento dos mecanismos responsáveis pelo aparecimento dos sintomas na dispepsia funcional. Além disso, novas drogas com capacidade potencial de interferir nestes mecanismos vêm sendo desenvolvidas. Estes fatos abrem a perspectiva de se dispor, no futuro próximo, de tratamento farmacológico mais racional e efetivo para a dispepsia funcional.

O objetivo deste artigo é prover breve revisão das novas drogas potencialmente úteis para o tratamento da dispepsia funcional. $\mathrm{O}$ entendimento dos mecanismos de ação destes medicamentos demanda considerar, inicialmente, alguns dos conhecimentos mais atuais sobre a origem dos sintomas nesta condição clínica.

\section{ORIGEM DOS SINTOMAS NA DISPEPSIA FUNCIONAL}

Os pacientes com dispepsia funcional podem se apresentar com sintomas variados, cuja expressão permite caracterizar diferentes tipos clínicos $^{(10)}$. De acordo com os novos critérios diagnósticos estabelecidos recentemente ("Critérios de Roma II") ${ }^{(41)}$, podem ser caracterizados três tipos principais: na dispepsia do tipo ulcerosa ("ulcer-like dyspepsia"), as queixas de dor epigástrica assemelham-se às da úlcera péptica. Na dispepsia motora ("dysmotility-like dyspepsia"), os sintomas de plenitude pós-prandial, saciedade precoce e, ocasionalmente, náuseas, sugerem que ocorram alterações da motilidade gastroduodenal. Em outro grupo de pacientes, predomina a queixa de pirose retroesternal, mas a tendência atual é a de classificar estes casos, que apresentam sintomas semelhantes aos da esofagite de refluxo ("reflux-like dyspepsia"), como portadores de doença funcional do esôfago ${ }^{(41)}$, que pode integrar o espectro da doença do refluxo gastroesofágico $^{(36)}$. Em muitos casos, sintomas semelhantes aos da úlcera péptica superpõe-se aos que sugerem alterações motoras, sem haver predominância clara de um ou outro grupo de manifestações. Isto caracteriza o tipo inespecífico da dispepsia funcional.

Os mecanismos que participam da origem dos sintomas na dispepsia funcional não são perfeitamente conhecidos. No entanto, três importantes fatores parecem estar envolvidos ${ }^{(26,41)}$ : a) anormalidades da motilidade gastrointestinal, b) aumento da sensibilidade a estímulos provenientes do lúmen do tubo digestivo e c) anormalidades da esfera psicoemocional.

As anormalidades da motilidade antro-piloro-duodenal constituem, provavelmente, o grupo de fatores etiopatogênicos que tem sido demonstrado há mais tempo e com maior freqüência na dispepsia funcional. Estudos efetuados já há algumas décadas têm demonstrado que pacientes com dispepsia funcional podem apresentar alterações da atividade mioelétrica gástrica ${ }^{(22)}$, redução da contratilidade antral ${ }^{(33)}$, incoordenação antro-piloro-duodenal e anormalidades da atividade motora duodeno-jejunal ${ }^{(23)}$. Estas alterações podem resultar em retardo do esvaziamento gástrico ${ }^{(4,33)}$, que ocorrem em cerca de metade dos casos de dispepsia funcional ${ }^{(14,26)}$.

Mais recentemente, outra anormalidade da motilidade gástrica $-\mathrm{a}$ diminuição da capacidade de acomodação do estômago - tem sido demonstrada de modo consistente em pacientes com dispepsia funcional, sobretudo naqueles em que predominam os sintomas de saciedade precoce e desconforto epigástrico pós-prandial. Embora a complacência gástrica não esteja particularmente alterada quando os pacientes são estudados em jejum ${ }^{(5,21)}$, a administração de uma refeiçãoteste é incapaz de provocar relaxamento significativo da porção proximal do estômago ${ }^{(37,45)}$, que é a região gástrica especializada para efetuar a acomodação do bolo alimentar ingerido ${ }^{(7)}$.

A deficiência de acomodação gástrica em pacientes com dispepsia motora pode associar-se à anormalidade da distribuição intragástrica do bolo alimentar, que parece ocorrer de forma independente do retardo do esvaziamento gástrico ${ }^{(44)}$. Nos pacientes dispépticos, diferentemente do que se verifica em pessoas normais, a maior parte da comida ingerida fica retida na parte mais distal do estômago, provocando distensão acentuada das suas paredes ${ }^{(4,18)}$ e denotando a já mencionada deficiência da função de reservatório do estômago proximal. Esta anormalidade pode, portanto, provocar sintomas pela estimulação de dois diferentes tipos de mecanorreceptores gástricos: 1) os sensíveis ao aumento anormal da pressão no fundus e 2) os sensíveis à distensão excessiva do antro gástrico.

Outro grupo de fatores etiopatogênicos de importância crescentemente reconhecida na dispepsia funcional, é o aumento da sensibilidade visceral. Esta anormalidade tem sido bem demonstrada pela 
constatação de que os dispépticos funcionais podem apresentar sintomas importantes, desencadeados pela distensão do estômago com volumes bem menores do que os que seriam necessários para causar qualquer tipo de sensação desagradável em pessoas normais ${ }^{(5)}$, ${ }^{21,28)}$. É importante notar que esta anormalidade parece restringir-se às vias sensoriais viscerais, uma vez que as medidas de tolerância a estímulos aplicados em órgãos de inervação do tipo somático, como a pele ou a musculatura esquelética, não revelam anormalidades ou sugerem, mesmo, que os pacientes funcionais parecem ter tolerância aumentada $^{(28)}$. É também interessante ressaltar que esta anormalidade sensorial não parece estar associada a alterações afetivas detectadas por testes psicométricos específicos ${ }^{(21)}$.

Adicionalmente, estudo mais recente demonstrou que pacientes com dispepsia funcional apresentam sensibilidade aumentada também à infusão de ácido no duodeno ${ }^{(34)}$. Esta anormalidade, por sua vez, ocasiona alteração da motilidade duodenal, que resulta em deficiência da remoção do ácido infundido, o que pode contribuir à manutenção do estímulo e, portanto, para a produção de sintomas ${ }^{(34)}$.

Um terceiro grupo de fatores etiopatogênicos é constituído pelos distúrbios psicológicos e emocionais. Há dados indicativos de maior prevalência, dentre os pacientes com dispepsia funcional, de antecedentes de dificuldades emocionais na infância ou na adolescência, de história prévia de abuso físico ou sexual e de anormalidades como ansiedade, depressão, hipocondria e neuroses ${ }^{(2,8,26,41)}$.

\section{NOVOS AGENTES TERAPÊUTICOS}

O tratamento farmacológico atual da dispepsia funcional compreende o uso empírico de anti-secretores, especialmente nos casos com sintomas semelhantes aos da úlcera péptica, e o emprego de agentes pró-cinéticos, para os pacientes com dispepsia tipo "dismotilidade" $(14,17,26,39,46)$. Na dispepsia tipo inespecífica, tanto os anti-secretores como os pró-cinéticos, bem como a combinação dos dois grupos de medicamentos, poderão trazer alívio dos sintomas.

O conjunto das novas drogas de aplicação potencial no tratamento da dispepsia funcional revisto no presente estudo, compreende agentes que visam a correção de distúrbios específicos demonstrados nesta condição e que exibem as seguintes ações farmacológicas: 1) aumento da contratilidade antral e da propulsão gastroduodenal; 2) relaxamento do estômago proximal e aumento da capacidade de acomodação do bolo alimentar e 3) diminuição da sensibilidade visceral ou modificação da percepção das sensações oriundas do tubo digestivo.

\section{Drogas que aumentam a propulsão gastroduodenal}

O conceito de que pacientes com dispepsia funcional podem apresentar diminuição anormal da contratilidade antral e retardo do esvaziamento gástrico, fundamenta o uso racional de drogas prócinéticas, como a metoclopramida, a cisaprida, a bromoprida e a domperidona ${ }^{(26)}$.

Estas drogas pró-cinéticas aumentam tanto a amplitude, como a freqüência das contrações antrais pós-prandiais e aceleram o esvaziamento gástrico ${ }^{(25)}$. Estes efeitos são conseqüentes à estimulação de receptores serotoninérgicos do tipo 4 (5-HT4) que, por sua vez, aumentam a liberação de acetilcolina de terminações nervosas do plexo mioentérico ${ }^{(25)}$

Novas drogas que apresentam efeitos semelhantes aos dos agentes pró-cinéticos incluem: a) novos agonistas 5-HT4 , b) antagonistas dos receptores A da colecistocinina (CCKA) e c) agonistas de receptores opióides. Dentre os novos agonistas 5-HT4, alguns agentes em desenvolvimento são benzamídicos, de estrutura molecular semelhante à da cisaprida e análogos ${ }^{(30)}$.

O antagonista CCKA melhor estudado é a loxiglumida, que apresenta efeito significativamente positivo sobre a contratilidade antral e o esvaziamento gástrico ${ }^{(16,35)}$

Dentre os novos agonistas opióides com potencial ação prócinética, deve ser destacada a trimebutina, droga já disponível há muitos anos no mercado brasileiro para o tratamento da síndrome do intestino irritável. A trimebutina exerce sua ação motora gastrointestinal pela estimulação não-seletiva de receptores opióides dos tipos mu, kappa e delta ${ }^{(20)}$. Novo e promissor agonista opióide é a fedotozina, um agente com afinidade seletiva para os receptores do tipo kappa, que parece agir tanto sobre a motilidade gástrica, como sobre a sensibilidade visceral ${ }^{(9,30)}$

Recente grupo de drogas com ação estimulatória sobre a contratilidade antral e o esvaziamento gástrico é o dos motilídios. Estas drogas, diferentemente dos pró-cinéticos, que agem sobre a motilidade digestiva pós-prandial, induzem o aparecimento de contrações semelhantes às da fase III do Complexo Motor Migrante Interdigestivo (CMMI). Este tipo particular de atividade motora é próprio dos períodos de jejum, sendo caracterizado por contrações vigorosas que se iniciam no antro gástrico e se propagam até o íleo. As contrações antrais da fase III do CMMI são as responsável pela remoção de resíduos não-digeríveis do interior do estômago ${ }^{(7)}$. Estas contrações se iniciam em resposta à ação da motilina, hormônio natural que age sobre receptores específicos localizados no plexo mientérico ${ }^{(7,47)}$. Os motilídios são, portanto, drogas que agem como agonistas dos receptores da motilina.

A primeira droga deste grupo de agentes motilídios a ter sua ação motora bem caracterizada foi um antibiótico macrolídio - a eritromicina ${ }^{(29,47)}$. Mais recentemente, foi relatado que outro antibiótico do mesmo grupo - a claritromicina - também possui ação motora análoga à da motilina ${ }^{(3)}$. Estudo recente de revisão ${ }^{(30)}$ registra que pelo menos três novos agonistas da motilina desprovidos de propriedades antibióticas acham-se em desenvolvimento. 


\section{Drogas que aumentam a acomodação gástrica}

Drogas de diferentes grupos farmacológicos podem provocar o relaxamento generalizado da musculatura gástrica, incluindo os nitratos $^{(19)}$ e os agonistas alfa-2 adrenégicos ${ }^{(43)}$. No entanto, estes agentes produzem o relaxamento não só da porção gástrica proximal, responsável pela acomodação do bolo alimentar, mas também a da região antro-piloro-duodenal. Isto acaba por provocar redução da contratilidade antral e inibição do esvaziamento gástrico, efeitos estes que se contrapõem ao eventual aumento da acomodação gástrica ocasionada por estes agentes.

Estudos do grupo de Leuven ${ }^{(11,37,38)}$, na Bélgica, vêm demonstrando que algumas drogas parecem induzir relaxamento predominante da região gástrica especializada na acomodação do bolo alimentar. Uma destas drogas é o sumatriptano, agente empregado no tratamento da enxaqueca, que se configura como um agonista dos receptores serotoninérgicos do tipo $1\left(5-\mathrm{HT}_{1}\right)$ do plexo mientérico, sem estimular os receptores $5-\mathrm{HT}_{3}$ e $5-\mathrm{HT}_{4}$

A injeção subcutânea do sumatriptano em pacientes com dispepsia funcional associa-se a significativo aumento do relaxamento fúndico induzido pela ingestão de uma refeição-teste, bem como da capacidade de acomodação gástrica do bolo alimentar ${ }^{(37)}$. Ainda que a administração do sumatriptano se associe à discreta inibição do esvaziamento gástrico, este efeito parece não interferir com o significativo aumento da capacidade de ingestão de uma refeição-teste líquida, antes que os sintomas pós-prandiais sobrevenham ${ }^{(37)}$.

Efeitos fundorrelaxantes semelhantes aos do sumatriptano foram obtidos com a administração de outro agonista 5 -HT - a buspirona $^{(11,}$ 38) -, que é uma droga de ação ansiolítica. Adicionalmente, pequeno ensaio terapêutico controlado com placebo, em pequena amostra de pacientes com dispepsia funcional, mostrou que a administração da buspirona (10 mg, três vezes ao dia) por 4 semanas produziu significativa melhora nos sintomas ${ }^{(38)}$

\section{Drogas que reduzem ou modificam a sensibilidade visceral}

Um conjunto bem diversificado de drogas de vários grupos farmacológicos, vem sendo avaliado quanto a sua capacidade de reduzir a percepção sensorial visceral em estudos feitos em animais, em voluntários normais e, sobretudo, em pacientes com distúrbios funcionais digestivos.

A octreotida, análogo sintético do hormônio natural somatostatina, em injeção subcutânea, aumenta significativamente o limiar para a percepção da distensão do intestino grosso em pacientes com a síndrome do intestino irritável ${ }^{(6)}$, sendo, porém, incerto se afeta a sensibilidade gastroduodenal em pacientes com a dispepsia funcional.

Os antagonistas CCK-A loxiglumida e seu isômero dexloxiglumida, ao lado de suas ações sobre a motilidade gastroduodenal, reduzem as sensações de repleção gástrica e de náusea induzidas pela infusão de lípides no duodeno ${ }^{(16,30,35)}$.

$\mathrm{O}$ antagonista seletivo de receptores 5-HT3 ondansetron parece aumentar de modo consistente o limiar de percepção de todo o tubo digestivo a estímulos variados, como a distensão das suas paredes ou a estimulação elétrica ${ }^{(24,30)}$. A administração oral do ondansetron foi capaz de reduzir a sensibilidade gástrica à distensão em voluntários normais ${ }^{(13,30)}$. Em ensaios terapêuticos com pacientes com a síndrome do intestino irritável, o ondansetron ocasionou melhora significativa de sintomas dispépticos associados ${ }^{(30)}$.

Novos antagonistas 5-HT3, como o granisetron e o alosetron, parecem ter efeitos semelhantes aos do ondansetron ${ }^{(30)}$, embora ainda não tenham sido especificamente testados quanto a sua capacidade de reduzir a sensibilidade visceral das porções superiores do tubo digestivo em pacientes dispépticos

Provavelmente, a droga mais promissora e melhor estudada deste grupo de agentes redutores da sensibilidade visceral seja a fedotozina. Este agonista seletivo dos receptores opióides kappa, ao lado das suas ações motoras e sobre a sensibilidade à distensão retal ${ }^{(30)}$, reduz significativamente a percepção sensorial associada à distensão do estômago $^{(9)}$. A ação da fedotozina sobre a sensibilidade visceral e a motilidade gastrointestinal parece ser exclusivamente periférica, sendo esta droga desprovida de efeitos sobre o sistema nervoso central ${ }^{(30)}$.

Vários ensaios terapêuticos controlados com placebo têm indicado a eficácia da fedotozina no tratamento de pacientes com a dispepsia funcional $^{(1,15,32)}$ e uma meta-análise de três destes estudos concluiu que esta droga pode ocasionar melhora significativa de quatro dos cinco sintomas dispépticos analisados ${ }^{(1)}$.

Um grupo de drogas crescentemente utilizadas em síndromes dolorosas crônicas e em pacientes com distúrbios funcionais digestivos é o dos antidepressivos tricíclicos ${ }^{(8,27,31)}$. É importante ressaltar que os eventuais efeitos benéficos destes agentes nestas condições clínicas têm sido observados com doses relativamente baixas, não sendo, portanto, atribuíveis às ações antidepressivas ou sedativas destas drogas, ou aos seus efeitos anticolinérgicos periféricos.

A amitriptilina em dose baixa (50 $\mathrm{mg}$ ao dia) produziu significativa melhora dos sintomas ${ }^{(27)}$ e dos índices de qualidade de $v$ ida $^{(31)}$ de pacientes com dispepsia funcional, em diferentes ensaios terapêuticos controlados com placebo. Porém, os mecanismos pelos quais este efeito benéfico se estabelece não são conhecidos. Esta droga parece não ocasionar modificação dos limiares de percepção da distensão do estômago com volumes crescentes ${ }^{(27)}$. Tendo em vista que a amitriptilina reduz a percepção da estimulação somática periférica, como por exemplo, a aplicação de corrente elétrica na pele dos braços, supõe-se que esta droga possa, de alguma forma análoga, aumentar a tolerância às sensações viscerais desagradáveis ${ }^{(30)}$.

Outro agente antidepressivo, a mianserina, parece ter efeito análogo ao dos tricíclicos, tendo sido demonstrado efeito terapêutico benéfico, 
significativamente superior ao do placebo, após 7 semanas de uso desta droga, em pacientes com várias síndromes funcionais digestivas, incluindo a dispepsia funcional ${ }^{(42)}$. É interessante salientar que neste estudo foram especificamente excluídos os casos com ansiedade ou transtornos afetivos $^{(8,42)}$.

\section{COMENTÁRIOS FINAIS}

O desenvolvimento de novas drogas, bem como a melhor caracterização da patogenia da dispepsia funcional, abrem perspectivas de tratamento farmacológico mais racional e eficaz desta condição clínica. No entanto, muitas das novas drogas que apresentam aplicabilidade potencial no tratamento da dispepsia funcional encontram-se, ainda, em desenvolvimento ou estão sendo testadas somente em estudos de base fisiopatológica ou em amostras pequenas de pacientes. Há, portanto, a óbvia necessidade de estudos controlados, com número adequado de casos apropriadamente selecionados, para que se possa concluir acerca da real utilidade destes novos agentes.

Por outro lado, ainda que o conhecimento sobre os mecanismos envolvidos na produção dos sintomas na dispepsia funcional (e abordáveis pelas novas drogas) tenha tido considerável expansão, permanece difícil saber quais os pacientes em que uma ou mais das alterações fisiopatológicas já demonstradas em trabalhos científicos está ou não presente. Isto ocorre, em grande parte, pelo fato de ser pequena a especificidade dos diferentes sintomas atribuíveis às porções superiores do tubo digestivo em indicar mecanismos fisiopatológicos subjacentes ${ }^{(2)}$. Possíveis exceções a esta regra são a pirose, que parece indicar inflamação ou estimulação da mucosa do esôfago distal ${ }^{(36,40,41)}$ e a saciedade precoce que, segundo observações recentes ${ }^{(37)}$, parece indicar com mais especificidade a deficiência de relaxamento do estômago proximal em resposta à alimentação. Além disso, os testes funcionais mais acurados, que têm sido utilizados para identificar a presença de anormalidades em dispépticos funcionais, permanecem restritos a centros mais especializados. Métodos que empregam equipamentos mais simples e mais difundidos, como a ultra-sonografia gástrica, adequada para não só caracterizar o perfil do esvaziamento gástrico $^{(4)}$, como também para avaliar outros aspectos relevantes da motilidade gástrica ${ }^{(18,19)}$, permanecem pouco utilizados.

Assim sendo, é previsível que a escolha dos novos agentes terapêuticos permanecerá, ainda por algum tempo, tendo base empírica. Neste sentido, favorece a sua aplicação terapêutica, mesmo empiricamente, o fato de que muitas das novas drogas, como, por exemplo, a loxiglumida ou a fedotozina agirem em mais de um mecanismo fisiopatológico, como a motilidade gastroduodenal e a percepção sensorial visceral ${ }^{(30)}$.

Por fim, é bem conhecido o fato de que, em ensaios terapêuticos controlados, a resposta satisfatória ao placebo ocorre em alto percentual de pacientes com a dispepsia funcional ${ }^{(26,39)}$. Isto indica que, além do princípio ativo do medicamento empregado, outros fatores parecem influir no sucesso do tratamento. Estes incluem a adequada orientação sobre a natureza da doença, a tranqüilização quanto à benignidade desta condição clínica, a garantia de acesso ao atendimento médico quando necessário e, sobretudo, a confiança no médico ${ }^{(12,26)}$. São aspectos que, independentemente da consecução dos objetivos previsíveis com o uso das novas drogas, deverão continuar a ser considerados no tratamento mais integral dos pacientes com a dispepsia funcional.

Troncon LEA. New drugs for the treatment of functional dyspepsia. Arq Gastroenterol 2001;38(3):207-212.

ABSTRACT - Background - Functional dyspepsia is defined by upper gastrointestinal symptoms without any evidence of structural abnormalities or organic disease. Current pharmacological treatment of functional dyspepsia is largely empirical and involves anti-secretory or prokinetic drugs. Aims - To review recent advances in the understanding of the mechanisms involved in symptom production in functional dyspepsia, as well as the development of new drugs that may interfere with these mechanisms, which may lead to more rational and effective treatment of this clinical condition. Method-Systematic review of papers published in English for the last 10 years. Results - New drugs that increase propulsive gastroduodenal motor activity include new benzamides similar to cisapride, CCK-A blockers, agonists of opiate receptors and motilin agonists similar to erythromycin. A number of agents, including sumatriptan and buspirone, stimulates serotonin receptors in the myoenteric plexuses and have been shown to increase gastric accommodation to a meal. Finally, a number of new drugs that either increase thresholds for visceral perception or modify sensations is currently under investigation. This includes agents of several groups, such as octreotide, loxiglumide, ondansetron and other serotonin blockers, fedotozine and tricyclic antidepressant at low doses. Conclusions - Although these new drugs may improve the pharmacological approach to the treatment of functional dyspepsia, there is a need for randomized, controlled trials to assess their efficacy. Moreover, difficulties related to the identification of the mechanisms underlying symptoms may limit the utilization of these new drugs.

HEADINGS - Dyspepsia, drug therapy. Intestinal diseases, physiopathology. Intestinal diseases, drug therapy. Drug therapy. Gastrointestinal motility. 


\section{REFERÊNCIAS BIBLIOGRÁFICAS}

1. Abitbol JL, Sherrer B, De Meynard C, Meric G, Fraitag B. Efficacy of fedotozine in functional dyspepsia: a meta-analysis of individual data from randomized, placebo-controlled studies [abstract]. Gut 1996;41:664-8.

2. Agréus L, Talley NJ. Dyspepsia: current understanding and management. Annu Rev Med 1997;49:475-93.

3. Annese V, Bortolotti M, Fiorella S, Conoscitore P, Perri F, Andriulli A. Clarithromycin accelerates gastric emptying by inducing powerful antral contractions [abstract]. Gastroenterology 2000;118(suppl. 2):A 623.

4. Bortolotti M, Bolondi, Santi V, Sarti P, Brunelli F, Barbara L. Patterns of gastric emptying in dysmotility-like dyspepsia. Scand J Gastroenterol 1995;30:408 10 .

5. Bradette M, Pare P, Douville P, Morin A. Visceral perception in health and functional dyspepsia. Crossover study of gastric distension with placebo and domperidone. Dig Dis Sci 1991;36:52-8.

6. Bradette M, Delvaux M, Staumont G, Fioramonti J, Bueno L, Frexinos J. Octreotide increases thresholds of colonic visceral perception in IBS patients without modifying muscle tone. Dig Dis Sci 1994:39:1171-8.

7. Camilleri M, Prather C. Gastric motor physiology and motor disorders. In: Feldman M, Sleisenger MH, Scharschmidt BF, editors. Gastrointestinal and liver disease. 6. ed. Philadelphia: W.B. Saunders; 1998. p.572-86.

8. Clouse RE. Psychotropic medications for the treatment of functional gastrointestinal disorders. Clin Perspect Gastroenterol 1999;2:348-56.

9. Coffin B, Bouhassira D, Chollet R, Fraitag B, De Meynard C, Geneve J, Lemann M, Willer JC, Jian R. Effect of the kappa agonist fedotozine on perception of gastric distension in healthy humans. Aliment Pharmacol Ther 1996;10:919-25.

10. Colin-Jones DG, Bloom B, Bodemar G, Crean G, Freston J, Gugler R. Management of dyspepsia: report of a working party. Lancet 1988;1:576-9.

11. Coulie B, Tack J, Janssens J. Influence of buspirone-induced fundus relaxation on the perception of gastric distension in man [abstract]. Gastroenterology 1997;112:A715.

12. Drossman DA. Diagnosing and treating patients with refractory functional gastrointestinal disorders. Ann Intern Med 1995;123:688-97.

13. Feinle C, Read NW. Ondansetron reduces nausea induced by gastroduodenal stimulation without changing gastric motility. Am J Physiol 1996;271:G591-7.

14. Fisher RS, Parkman HP. Management of nonulcer dyspepsia. N Engl J Med 1998;339:1376-81.

15. Fraitag B, Homerin M, Hecketsweller P. Double-blind dose-response multicenter comparison of fedotozine and placebo in treatment of nonulcer dyspepsia. Dig Dis Sci 1994:39:1072-7.

16. Fried M, Erlacher U, Schwizer W, Lochner C, Koerfer J, Beglinger C, Jansen JB, Lamers CB, Harder F, Bischof-Delaloye A, Stadler GA, Rovati L. Role of cholecystokinin in the regulation of gastric emptying and pancreatic enzyme secretion in humans. Studies with the cholecystokinin-receptor antagonis loxiglumide. Gastroenterology 1991;101:503-11.

17. Halter F, Miazza B, Brignoli R. Cisapride or cimetidine in the treatment of functional dyspepsia. Scand J Gastroenterol 1994;29:618-23.

18. Hausken T, Berstad A. Wide gastric antrum in patients with non-ulcer dyspepsia: effect of cisapride. Scand J Gastroenterol 1992;27:427-32.

19. Hausken T, Berstad A. Effect of glyceryl trinitrate on antral motility and symptoms in patients with functional dyspepsia. Scand J Gastroenterol 1994;29:23-8.

20. Kamiya T, Nagao T, Andouu T, Misu N, Kobayashy Y, Kirako M, Hara M., Fujinam T. Effects of trimebutine maleate on gastric motility in patients with gastric ulcer. J Gastroenterol 1998;33:823-7.

21. Lémann M, Dederding JP, Flourié B, Franchisser C, Rambaud JC, Jian R. Abnormal perception of visceral pain in response to gastric distension in chronic idiopathic dyspepsia. Dig Dis Sci 1991;36:1249-54.

22. Lin Z, Eaker EY, Sarosiek I, McCallum RW. Gastric myoelectrical activity and gastric emptying in patients with functional dyspepsia. Am J Gastroenterol 1999;94:2384-9

23. Malagelada JR, Stanghellini V. Manometric evaluation of functional upper gut symptoms. Gastroenterology 1985;88:1223-31.

24. Maxton DG, Morris J, Whorwell PJ. Selective 5-hydroxytryptamine antagonism: a role in irritable bowel syndrome and functional dyspepsia? Aliment Pharmacol Ther 1996;10:595-9.
25. McCallum RW. Cisapride: a new class of prokinetic agent. The ACG committee on FDA-related matters. American College of Gastroenterology. Am J Gastroenterol 1991;86:135-49.

26. McQuaid K. Dyspepsia. In: Feldman M, Sleisenger MH, Scharschmidtd BF, editors. Gastrointestinal and liver disease. 6. ed. Philadelphia; W.B. Saunders: 1998. p.105-17.

27. Mertz H, Fass R, Kodner A, Yan-Go F, Fullerton S, Mayer EA. Effect of amitryptiline on symptoms, sleep and visceral perception in patients with functional dyspepsia Am J Gastroenterol 1998;93:160-5

28. Mertz H, Fullerton S, Naliboff B, Meyer EA. Symptoms and visceral perception in severe functional and organic dyspepsia. Gut 1998;42:814-22.

29. Otterson MF, Sarna SK. Gastrointestinal motor effects of erythromycin. Am J Physiol 1990;259:G355-63.

30. Pandolfino JE, Howden CW, Kahrilas PJ. Motility-modifying agents and management of disorders of gastrointestinal motility. Gastroenterology 2000;118(Suppl 1):S32-47.

31. Randall CW. Amitryptiline improves the quality of life in patients with nonulcer dyspepsia [abstract)]. Gastroenterology 1998;114:A265.

32. Read NW, Abitbol JL, Bardhan KD, Whorwell PJ, Fraitag B. Efficacy and safety of the peripheral kappa agonist fedotozine versus placebo in the treatment of functional dyspepsia. Gut 1997;41:664-8.

33. Rees WDW, Miller LJ, Malagelada JR. Dyspepsia, antral motor dysfunction, and gastric stasis of solids. Gastroenterology 1980;78:360-5.

34. Samsom M, Verhagen MA, Vanberge-Henegouwen GP, Smout AJ. Abnormal clearance of exogenous acid and increased acid sensitivity of the proximal duodenum in dyspeptic patients. Gastroenterology 1999;116:515-20.

35. Schwizer E, Borovicka J, Kunz P, Fraser R, Kreiss C, D'amato M, Creller G, Boesiger P, Fried M. Role of cholecystokinin in the regulation of liquid gastric emptying and gastric motility on humans: studies with the CCK antagonist loxiglumide. Gut 1997:41:500-4

36. Sonnenberg A, Delco F, El-Serag H. Empiric therapy versus diagnostic tests in gastroesophageal reflux disease. A medical decision analysis. Dig Dis Sci 1998:43:1001-8.

37. Tack J, Piessevaux H, Coulie B, Caenepeel P, Janssens J. Role of impaired gastric accommodation to a meal in functional dyspepsia. Gastroenterology 1998; $115: 1346-52$.

38. Tack J, Piessevaux H, Coulie B, Fischler B, De Gucht V, Janssens J. A placebocontrolled trial of buspirone, a fundus relaxing drug, in functional dyspepsia: effects on symptoms and gastric sensory and motor function [abstract]. Gastroenterology 1999;116:A325.

39. Talley NJ. Review article: functional dyspepsia - should treatment be targeted on disturbed physiology? Aliment Pharmacol Ther 1995;9:107-15.

40. Talley NJ, Silverstein MD, Agreus L Nyren O, Sonnenberg A, Holtman G. AGA technical review: evaluation of dyspepsia. Gastroenterology 1998;114:58295.

41. Talley NJ, Stanghellini V, Heading RC, Koch Kl, Malagelada JR, Tytgat GNJ. Functional gastroduodenal disorders. Gut 1999;45(Suppl II):II37- 42.

42. Tanum L, Malt UF. A new pharmacologic treatment of functional gastrointestina disorder. A double-blind placebo-controlled study with mianserin. Scand J Gastroenterol 1996;31:318-25

43. Thumshirn M, Camilleri M, Choi MG, Zinsmeister AR. Modulation of gastric sensory and motor functions by mitrergic and alpha2-adrenergic agents in humans. Gastroenterology 1999;116:573-85.

44. Troncon LEA, Bennett RJM, Thompson DG, Ahluwalia NK. Abnormal distribution of food in dysmotility-like functional dyspepsia. Gut 1994;35:32733.

45. Troncon LEA, Thompson DG, Ahluwalia NK, Barlow J, Heggie LJ. Relationship between upper abdominal symptoms and gastric distension in dysmotility-like functional dyspepsia and post-vagotomy patients. Gut 1995:37:17-23.

46. Veldhuysen Van Zenten SJ, Cleary C, Talley NJ, Peterson TC, Nyren O, Bradley LA, Verlinden M, Tytgat GN. Drug treatment of functional dyspepsia. Am J Gastroenterol 1996;91:660-73.

47. Weber FJ Jr, Richards RD, McCallum RW. Erythromycin: a motilin agonist and gastrointestinal and pro-cinetic agent. Am J Gastroenterol 1993; 88:485-90.

Recebido em 29/11/2000 Aprovado em 13/3/2001 